\title{
Black hole thermodynamics and two-dimensional dilaton gravity theory
}

\author{
Donam Youm* \\ Theory Division, CERN, CH-1211, Geneva 23, Switzerland \\ (Received 2 November 1999; published 27 January 2000)
}

\begin{abstract}
We relate various black hole solutions in the near-horizon region to black hole solutions in two-dimensional dilaton gravity theories in order to argue that the thermodynamics of black holes in $D \geqslant 4$ can be effectively described by the thermodynamics of black holes in two-dimensional dilaton gravity theories. We show that the Bekenstein-Hawking entropies of single-charged dilatonic black holes and dilatonic $p$ branes with an arbitrary dilaton coupling parameter in arbitrary spacetime dimensions are exactly reproduced by the BekensteinHawking entropy of the two-dimensional black hole in the associated two-dimensional dilaton gravity model. We comment that the thermodynamics of a nonextreme stringy four-dimensional black hole with four charges and a five-dimensional black hole with three charges may be effectively described by the thermodynamics of black hole solutions with a constant dilaton field in two-dimensional dilaton gravity theories.
\end{abstract}

PACS number(s): 04.70.Dy, 04.50.+h, 04.70.Bw, 11.25.Mj

\section{INTRODUCTION}

The recent development in string duality and the observation [1] that D branes can carry charges of form potentials in the Ramond-Ramond (RR) sector of string theories enabled us to address fundamental issues in quantum gravity such as the statistical interpretation of black hole entropy within the framework of string theory. By applying the D-brane counting technique, pioneered in Ref. [2], the BekensteinHawking entropies of black hole solutions in string theories have been successfully reproduced. Generally, the D-brane counting technique can be applied to the Bogomol'nyiPrasad-Sommerfield (BPS) and the near-BPS cases and a few other special cases such as a non-BPS extreme rotating black hole in four dimensions.

The holographic principle [3-5] also has contributed to the microscopic interpretation of the Bekenstein-Hawking entropy. Brown and Henneaux [6] showed that the asymptotic symmetry group of $\mathrm{AdS}_{3}$ space is the conformal group in two-dimensional spacetime at the boundary. By using this idea, Carlip $[7,8]$ and Strominger [9] were able to exactly reproduce the Bekenstein-Hawking entropy of the three-dimensional constant curvature black hole of Banãdos, Teitelboim, and Zanelli (BTZ) [10] by counting microscopic degrees of freedom of the conformal field theory at the boundary. An important observation [11] by Hyun that (uplifted) black hole solutions in string theory can be put in the form of the product of the BTZ black hole and a sphere through a series of $U$-duality transformations, enabled the microscopic counting of nonextreme stringy black holes in four and five dimensions by applying the result of Carlip and Strominger (for example, Ref. [12]).

As pointed out in Ref. [11], higher-dimensional black hole solutions in string theories can also be related to twodimensional black hole solutions through $U$-duality transformations. The four- and the five-dimensional nonextreme black holes in string theory are related [13-17] to twodimensional black holes in the Jackiw-Teitelboim (JT) model

\footnotetext{
*Email address: Donam.Youm@cern.ch
}

$[18,19]$ and the Callan-Giddings-Harvey-Strominger (CGHS) model [20] and to the two-dimensional charged black hole of McGuigan, Nappi, and Yost [21]. Recently, progress has been made [22-24] in reproducing the Bekenstein-Hawking entropies of the two-dimensional black holes by using Cardy's formula [25] for the boundary conformal theory. Furthermore, it is argued in Refs. $[26,27]$ that the Bekenstein-Hawking entropy of the generic black holes in arbitrary dimensional pure gravity theories can be reproduced by the microscopic calculation based on the conformal theory associated with the two-dimensional subset of the spacetime. So, it seems that black holes in two-dimensional gravity theories, which were originally studied as unrealistic toy models for the quantum theory of gravity, have a closer connection with realistic black holes in $D \geqslant 4$ than it was originally thought.

It is the purpose of this paper to relate various black hole solutions in $D \geqslant 4$ to black hole solutions in two-dimensional dilaton gravity theories. In Sec. II, we relate a single-charged dilatonic black hole solution in $D \geqslant 4$ with an arbitrary dilaton coupling parameter to a black hole solution in a twodimensional dilaton gravity theory. We find that the Bekenstein-Hawking entropies of these two black holes are the same in the large charge limit or the near-extremal limit. In Sec. III, we argue that the thermodynamics of a dilatonic $p$ brane with an arbitrary dilaton couple parameter in arbitrary spacetime dimensions can also be described by a black hole solution in a two-dimensional dilaton gravity theory. In Sec. IV, we comment that thermodynamics of the nonextreme four-dimensional stringy black hole with four charges and the nonextreme five-dimensional stringy black hole with three charges may be more naturally described by the black holes with constant dilaton field in two-dimensional dilaton gravity theories.

\section{SINGLE-CHARGED DILATONIC BLACK HOLES}

In this section, we consider single-charged dilatonic black hole solutions with an arbitrary dilaton coupling parameter $a$ in arbitrary spacetime dimensions $D$. The result in this section can be applied not only to all the single-charged dila- 
tonic black holes but also to multicharged black holes in string theories, because in the near-horizon limit the actions for the multicharged black hole solutions in string theories can be reduced (with the guidance of the explicit solutions in the near-horizon limit) to the actions for the two-dimensional dilaton gravity with possibly different forms of the dilaton potential term from the one obtained in this section. The exceptional case is the multicharged stringy black hole solutions with a regular BPS limit, i.e., the four-dimensional black hole with four charges and the five-dimensional black hole with three charges. In this special case, all the scalars of the solution become constant in the near-horizon limit and therefore one cannot relate such black hole solutions to black hole solutions with a nontrivial dilaton field in twodimensional dilaton gravity models. ${ }^{1}$ This exceptional case will be separately discussed in the last section.

The corresponding Einstein-frame action is

$$
S_{E}=\frac{1}{2 \kappa_{D}^{2}} \int d^{D} x \sqrt{-G^{E}}\left[\mathcal{R}_{G^{E}}-\frac{4}{D-2}(\partial \phi)^{2}-\frac{1}{4} e^{2 a \phi} F_{2}^{2}\right],
$$

where $\kappa_{D}$ is the $D$-dimensional Einstein gravitational constant and $F_{2}$ is the field strength of the U(1) gauge potential $A^{(1)}=A_{M} d x^{M}(M=0,1, \ldots, D-1)$. The nonextreme black hole solution to the field equations of this action is given by

$$
\begin{aligned}
d s_{E}^{2}= & -H^{-4(D-3) /(D-2) \Delta} f d t^{2}+H^{4 /(D-2) \Delta}\left[f^{-1} d r^{2}\right. \\
& \left.+r^{2} d \Omega_{D-2}^{2}\right], \\
e^{\phi}= & H^{(D-2) a / 2 \Delta}, \quad A_{t}=-H^{-1},
\end{aligned}
$$

where

$$
\begin{aligned}
& H=1+\frac{m \sinh ^{2} \alpha}{r^{D-3}}, \quad f=1-\frac{m}{r^{D-3}}, \\
& \Delta=\frac{(D-2) a^{2}}{2}+\frac{2(D-3)}{D-2} .
\end{aligned}
$$

The Bekenstein-Hawking entropy $S_{B H}$ of the dilatonic black hole solution (2) is determined by the surface area $A_{H}$ of the event horizon (located at $r=r_{H}=m^{1 /(D-3)}$ ):

\footnotetext{
${ }^{1}$ On the other hand, the near-horizon region solutions for this case can be related to the BTZ black hole solution when the solutions are uplifted to one higher spacetime dimensions, after the U-duality transformations when necessary. (Note, however, that in such embeddings of black hole solutions the harmonic function for the charge associated with the gravitational wave is allowed to not take the near-horizon form and therefore some of scalars are not constant.) The action for the BTZ black hole solution can be reduced to a two-dimensional dilaton gravity model action upon dimensional reduction. But in this paper, we ignore the higher-dimensional origin of the black hole solution, just considering the Einstein-frame metric itself. See the second paragraph of the last section for more comments.
}

$$
S_{B H}=\frac{A_{H}}{4 G_{D}}=\frac{m^{(D-2) /(D-3)} V_{S^{D-2}} \cosh ^{4 / \Delta} \alpha}{4 G_{D}},
$$

where $V_{S^{D-2}}=2 \pi^{(D-2) / 2} / \Gamma[(D-1) / 2]$ is the volume of $S^{D-2}$ with the unit radius.

One can also think of the dilatonic black hole in $D$ dimensions as being magnetically charged under the $(D-2)$-form field strength $F_{D-2}$. The corresponding Einstein-frame action is

$$
\begin{aligned}
\widetilde{S}_{E}= & \frac{1}{2 \kappa_{D}^{2}} \int d^{D} x \sqrt{-G^{E}}\left[\mathcal{R}_{G^{E}}-\frac{4}{D-2}(\partial \phi)^{2}\right. \\
& \left.-\frac{1}{2 \cdot(D-2) !} e^{-2 a \phi} F_{D-2}^{2}\right] .
\end{aligned}
$$

In terms of this "dual" field parametrization, the dilatonic black hole solution takes the following form:

$$
\begin{aligned}
d s_{E}^{2}= & -H^{-4(D-3) /(D-2) \Delta} f d t^{2}+H^{4 /(D-2) \Delta}\left[f^{-1} d r^{2}\right. \\
& \left.+r^{2} d \Omega_{D-2}^{2}\right], \\
e^{\phi}= & H^{(D-2) a / 2 \Delta}, \quad F_{D-2}=\star(d H \wedge d t) .
\end{aligned}
$$

In the so-called dual-frame [28-31], the spacetime of the BPS dilatonic black hole in the near-horizon region takes the $\mathrm{AdS}_{2} \times S^{D-2}$ form. The following dual-frame action is related to Eq. (5) through the Weyl rescaling transformation $G_{M N}^{E}=e^{-2 a \phi /(D-3)} G_{M N}^{d}$ :

$$
\begin{aligned}
\widetilde{S}_{d}= & \frac{1}{2 \kappa_{D}^{2}} \int d^{D} x \sqrt{-G^{d}} e^{\delta \phi}\left[\mathcal{R}_{G^{d}}+\gamma(\partial \phi)^{2}\right. \\
& \left.-\frac{1}{2 \cdot(D-2) !} F_{D-2}^{2}\right]
\end{aligned}
$$

where the parameters in the action are defined as

$$
\delta \equiv-\frac{D-2}{D-3} a, \quad \gamma \equiv \frac{D-1}{D-2} \delta^{2}-\frac{4}{D-2} .
$$

In the dual frame, the dilatonic black hole solution takes the following form:

$$
\begin{aligned}
d x_{d}^{2}= & -H^{[2 \Delta-4(D-3)] /[(D-3) \Delta]} f d t^{2}+H^{2 /(D-3)}\left[f^{-1} d r^{2}\right. \\
& \left.+r^{2} d \Omega_{D-2}^{2}\right],
\end{aligned}
$$

where the dilaton and the $(D-2)$-form field strength take the same forms as in Eq. (6). In the near-horizon region, the metric (9) is approximated to

$$
\begin{aligned}
d s_{d}^{2} \approx & -\left(\frac{\hat{\mu}}{r}\right)^{2-[4(D-3) / \Delta]}\left(1-\frac{m}{r^{D-3}}\right) d t^{2} \\
& +\left(\frac{\hat{\mu}}{r}\right)^{2}\left(1-\frac{m}{r^{D-3}}\right)^{-1} d r^{2}+\hat{\mu}^{2} d \Omega_{D-2}^{2},
\end{aligned}
$$


where $\hat{\mu} \equiv\left(m \sinh ^{2} \alpha\right)^{1 /(D-3)}$, and the dilaton and the nonzero component of the U(1) gauge field are approximated to

$$
e^{\phi} \approx\left(\frac{\hat{\mu}}{r}\right)^{(D-2)(D-3) a / 2 \Delta}, \quad A_{t} \approx\left(\frac{r}{\hat{\mu}}\right)^{D-3}
$$

By compactifying the dual-frame action (7) on $S^{D-2}$ with the radius $\hat{\mu}$, one obtains the following two-dimensional effective action:

$$
S=\frac{1}{2 \kappa_{2}^{2}} \int d^{2} x \sqrt{-g} e^{\delta \phi}\left[\mathcal{R}_{g}+\gamma(\partial \phi)^{2}+\Lambda\right],
$$

where the $\kappa_{2}^{2}$ is the two-dimensional gravitational constant and the cosmological constant $\Lambda$ is given by

$$
\Lambda=\frac{D-3}{2 \hat{\mu}^{2}}\left[2(D-2)-\frac{4(D-3)}{\Delta}\right] .
$$

To bring the action (12) to the standard form of the twodimensional dilaton gravity action, one redefines the dilaton as $\Phi=e^{\delta \phi}$ and then applies the Weyl rescaling of the metric $g_{\mu \nu}=\Phi^{-\gamma / \delta^{2}} e^{\Phi / 2} \widetilde{g}_{\mu \nu}$. The resulting action has the following form [32]:

$$
S=\frac{1}{2 \kappa_{2}^{2}} \int d^{2} x \sqrt{-\tilde{g}}\left[\Phi \mathcal{R}_{g}+\frac{1}{2} \partial_{\mu} \Phi \partial^{\mu} \Phi+\Lambda \Phi^{1-\gamma / \delta^{2}} e^{\Phi / 2}\right]
$$

The kinetic term for the dilaton $\Phi$ can be removed by applying one more Weyl rescaling $\tilde{g}_{\mu \nu}=e^{-\Phi / 2} \bar{g}_{\mu \nu}$, resulting in the following action [32]:

$$
S=\frac{1}{2 \kappa_{2}^{2}} \int d^{2} x \sqrt{-\bar{g}}\left[\Phi \mathcal{R}_{g}^{-}+\Phi^{1-\gamma / \delta^{2}} \Lambda\right] .
$$

Particularly interesting special cases of this action are the JT model for the $(D, a)=(4,1 / \sqrt{3})$ case and the CGHS model for the $(D, a)=(4,1)$ case.

The field equations of the action (15) are

$$
\begin{gathered}
\mathcal{R}_{g}+\left(1-\frac{\gamma}{\delta^{2}}\right) \Phi^{-\gamma / \delta^{2}} \Lambda=0, \\
\nabla_{\mu} \nabla_{\nu} \Phi-\bar{g}_{\mu \nu} 1 / 2 \Phi^{1-\gamma / \delta^{2}} \Lambda=0 .
\end{gathered}
$$

In the Schwarzschild gauge, the general time-dependent solution to these field equations takes the following form: ${ }^{2}$

\footnotetext{
${ }^{2}$ The most general solution in two-dimensional dilaton gravity theory with general dilaton potential was previously constructed in Ref. [33].
}

$$
\begin{aligned}
d s^{2}= & -\left[\frac{\delta^{2}}{2 \delta^{2}-\gamma}\left(\frac{x}{l}\right)^{2-\gamma / \delta^{2}}-2 l M\right] d \tau^{2} \\
& +\left[\frac{\delta^{2}}{2 \delta^{2}-\gamma}\left(\frac{x}{l}\right)^{2-\gamma / \delta^{2}}-2 l M\right]^{-1} d x^{2}, \\
\Phi & =\frac{x}{l},
\end{aligned}
$$

where $l \equiv 1 / \sqrt{\Lambda}$ and the diffeomorphism invariant parameter $M$ [34] defined in the following is the mass of the solution:

$$
M=-\frac{1}{2 l}\left[(\nabla \Phi)^{2} l^{2}+\frac{\delta^{2}}{2 \delta^{2}-\gamma} \Phi^{2-\gamma / \delta^{2}}\right] .
$$

The thermodynamic properties of the solution (17) is determined by the behavior of the solution at the event horizon. The event horizon is located at the root of $\bar{g}_{\tau \tau}\left(\Phi_{H}\right)=0$, namely at

$$
\Phi_{H}=\left[2 l M\left(2-\frac{\gamma}{\delta^{2}}\right)\right]^{\delta^{2} /\left(2 \delta^{2}-\gamma\right)}
$$

At the event horizon, the Killing vector $k^{\mu}=l \eta^{\mu \nu} \Phi_{, \nu}$ is null due to the definition (18) of the mass $M$ :

$$
\begin{aligned}
\left.|k|^{2}\right|_{\Phi_{H}} & =-\left.l^{2}|\nabla \Phi|^{2}\right|_{\Phi_{H}}=2 l M-\left.\frac{\delta^{2}}{2 \delta^{2}-\gamma} \Phi^{2-\gamma / \delta^{2}}\right|_{\Phi_{H}} \\
& =\bar{g}_{\tau \tau}\left(\Phi_{H}\right)=0 .
\end{aligned}
$$

The surface gravity $\kappa$, which determines the Hawking temperature $T_{H}=\kappa / 2 \pi$ and is defined by $\kappa^{2}=$ $-\left.\frac{1}{2} \nabla^{\mu} k^{\nu} \nabla_{\mu} k_{\nu}\right|_{\Phi_{H}}$, is given by

$$
\kappa=\frac{1}{2 l} \Phi_{H}^{1-\gamma / \delta^{2}}=\frac{1}{2 l}\left[2 l M\left(2-\frac{\gamma}{\delta^{2}}\right)\right]^{\left(\delta^{2}-\gamma\right) /\left(2 \delta^{2}-\gamma\right)}
$$

Given the above expressions for the mass $M$ and the surface gravity $\kappa$ of the solution, one can see by using the first law of the thermodynamics that the Bekenstein-Hawking entropy is

$$
S_{B H}=\frac{2 \pi}{\kappa_{2}^{2}} \Phi_{H}=\frac{2 \pi}{\kappa_{2}^{2}}\left[2 l M\left(2-\frac{\gamma}{\delta^{2}}\right)\right]^{\delta^{2} /\left(2 \delta^{2}-\gamma\right)} .
$$

We now show that the Bekenstein-Hawking entropy (22) of the two-dimensional black hole compactified from the dilatonic black hole in $D \geqslant 4$ is the same as the BekensteinHawking entropy (4) of the original dilatonic black hole in $D \geqslant 4$. One can bring the two-dimensional part $g_{\mu \nu}=G_{\mu \nu}^{d}$ ( $\mu, \nu=t, r)$ of the near-horizon metric (10) to the form (17) of the solution of the two-dimensional dilaton gravity theory 
with the action (15) by applying the Weyl rescaling $g_{\mu \nu}$ $=\Phi^{-\gamma / \delta^{2}} \bar{g}_{\mu \nu}=e^{-\gamma / \delta} \bar{g}_{\mu \nu}$ and then redefining the coordinates in the following way:

$$
t=\frac{|\delta|}{\sqrt{2 \delta^{2}-\gamma}} \tau, \quad r=\hat{\mu}\left(\frac{x}{l}\right)^{2 \Delta /\left[(D-2)^{2} a^{2}\right]}
$$

The resulting metric $\bar{g}_{\mu \nu}$ has the form (17) with the mass $M$ given by

$$
M=\frac{\delta^{2}}{2 l\left(2 \delta^{2}-\gamma\right) \sinh ^{2} \alpha} .
$$

By plugging the expression (24) for the mass $M$ into the expression (22) for the entropy of the two-dimensional solution (17), making use of the following relation:

$$
\begin{aligned}
\kappa_{2}^{2} & =\frac{\kappa_{D}^{2}}{\hat{\mu}^{D-2} V_{S^{D-2}}} \\
& =\frac{\kappa_{D}^{2}}{m^{(D-2) /(D-3)} \sinh ^{2(D-2) /(D-3)} \alpha V_{S^{D-2}}},
\end{aligned}
$$

one obtains the following expression for the entropy:

$$
\begin{aligned}
S_{B H} & =\frac{2 \pi}{\kappa_{2}^{2}}\left(\frac{1}{\sinh ^{2} \alpha}\right)^{\delta^{2} /\left(2 \delta^{2}-\gamma\right)} \\
& =\frac{2 \pi}{\kappa_{D}^{2}} m^{(D-2) /(D-3)} V_{S^{D-2} \sinh ^{4 / \Delta} \alpha .}
\end{aligned}
$$

Note, the Einstein gravitational constant $\kappa_{D}$ is related to the Newton constant $G_{D}$ as $\kappa_{D}^{2}=8 \pi G_{D}$ in the unit $c=1$. So, in the limit of large $\alpha$ (i.e., the large charge limit or the nearextremal limit), in which $\sinh \delta \approx \cosh \delta$, the BekensteinHawking entropy (26) of the two-dimensional solution (17) with Eq. (24) becomes exactly the same as the BekensteinHawking entropy (4) of the $D$-dimensional dilatonic black hole (2). Therefore, thermodynamics of the $D$-dimensional dilatonic black hole solution (2) can be effectively described by the thermodynamics of the two-dimensional black hole solution (17).

\section{DILATONIC $p$ BRANES}

When all the longitudinal directions are compactified on a compact manifold, a $p$ brane in $D$ spacetime dimensions re- duces to a dilatonic black hole in $D-p$ spacetime dimensions. So, by using the result of the previous section, one can see that thermodynamics of dilatonic $p$ branes can also be effectively described by black holes in two-dimensional dilaton gravity models. Also, the thermodynamics of the delocalized intersecting brane solutions can be described by the thermodynamics of black holes in two-dimensional dilaton gravity theories, since all the delocalized intersecting brane solutions reduce to multicharged black holes after all the longitudinal and the relative transverse directions are compactified. In the following, we relate a dilatonic $p$ brane in $D$ spacetime dimensions to a dilatonic black hole in $D-p$ spacetime dimensions.

The Einstein-frame action for the $D$-dimensional dilatonic $p$ brane with an arbitrary dilaton coupling parameter $b$ is given by

$$
\begin{aligned}
S_{E}^{p}= & \frac{1}{2 \kappa_{D}^{2}} \int d^{D} x \sqrt{-G^{E}}\left[\mathcal{R}_{G^{E}}-\frac{4}{D-2}(\partial \phi)^{2}\right. \\
& \left.-\frac{1}{2 \cdot(p+2) !} e^{2 b \phi} F_{p+2}^{2}\right],
\end{aligned}
$$

where $F_{p+2}$ is the field strength of the $(p+1)$-form potential $A^{(p+1)}=A_{M_{1}} \ldots M_{p+1} d x^{M_{1}} \wedge \ldots \wedge d x^{M_{p+1}}\left(M_{1}, \ldots, M_{p+1}\right.$ $=0,1, \ldots, D-1)$. The nonextreme dilatonic $p$-brane solution to the field equations of this action has the following form:

$$
\begin{aligned}
d s_{E}^{2}= & H_{p}^{-4(D-p-3) /\left[(D-2) \Delta_{p}\right]}\left[-f_{p} d t^{2}+d x_{1}^{2}+\cdots+d x_{p}^{2}\right] \\
& +H_{p}^{4(p+1) /\left[(D-2) \Delta_{p}\right]}\left[f_{p}^{-1} d r^{2}+r^{2} d \Omega_{D-p-2}^{2}\right] \\
e^{\hat{\phi}}= & H_{p}^{(D-2) b /\left(2 \Delta_{p}\right)}, \quad A_{t x_{1} \ldots x_{p}}=-H_{p}^{-1}
\end{aligned}
$$

where

$$
\begin{aligned}
& H_{p}=1+\frac{m \sinh ^{2} \alpha_{p}}{r^{D-p-3}}, \quad f_{p}=1-\frac{m}{r^{D-p-3}}, \\
& \Delta_{p}=\frac{(D-2) b^{2}}{2}+\frac{2(p+1)(D-p-3)}{D-2} .
\end{aligned}
$$

The Bekenstein-Hawking entropy $S_{B H}$ of the dilatonic $p$-brane solution (28) is determined by the surface area $A_{H}$ of the horizon (located at $\left.r=r_{H}=m^{1 /(D-p-3)}\right)$ :

$$
S_{B H}=\frac{A_{H}}{4 G_{D}}=\frac{m^{(D-p-2) /(D-p-3)} V_{S^{D-p-2}} \cosh ^{4(p+1)(D-p-2) /\left[(D-2) \Delta_{p}\right]} \alpha_{p}}{4 G_{D}} .
$$


Since the $p$-brane solution (28) does not depend on the longitudinal coordinates $x_{i}(i=1, \ldots, p)$, i.e., has the isometry along these directions, one can compactify the solution along the longitudinal directions on $T^{p}$ to obtain a black hole solution in $D-p$ spacetime dimensions. Such dimensional reduction of the dilatonic $p$-brane solution (28) leads to a dilatonic black hole solution of the form (2) in $D-p$ spacetime dimensions (i.e., $D$ in the solution (2) is replaced by $D-p$ ) with the dilaton coupling parameter $a$ given by

$$
a=\sqrt{\frac{D-p}{D-p-2} b^{2}+\frac{4(D-p-3)^{2} p}{(D-2)(D-p-2)^{2}}} .
$$

The Bekenstein-Hawking entropy of such a black hole solution obtained from the dilatonic $p$ brane through the dimensional reduction procedure has the following form:

$$
S_{B H}=\frac{A_{H}}{4 G_{D-p}}=\frac{m^{(D-p-2) /(D-p-3)} V_{S^{D-p-2}} \cosh ^{4 / \Delta} \alpha_{p}}{4 G_{D-p}},
$$

where $\Delta$ is given by Eq. (3) with $D$ replaced by $D-p$ and $a$ given by Eq. (31). By using the fact that the value of $\Delta_{p}$ does not change under the dimensional reduction $\left[\mathrm{so}, \Delta_{p}\right.$ in Eq. (30) and $\Delta$ in Eq. (32) are the same] and the following relation between the $D$-dimensional Newton constant $G_{D}$ and the $(D-p)$-dimensional Newton constant $G_{D-p}$ :

$$
G_{D-p}=\frac{G_{D}}{V_{T^{p}}}=\frac{G_{D}}{\cosh ^{4 p(D-p-3) /\left[(D-2) \Delta_{p}\right]} \alpha_{p}},
$$

where $V_{T^{p}}$ is the volume of $T^{p}$, on which the dilatonic $p$ brane is compactified, one can see that the entropy (32) of the dimensionally reduced black hole in $(D-p)$ dimensions is the same as the entropy (30) of the dilatonic $p$ brane (28) in $D$ dimensions. In the previous section, we have shown that the Bekenstein-Hawking entropy of the dilatonic black hole is the same as the Bekenstein-Hawking entropy of the corresponding two-dimensional solution in the limit of large charge or the near-extremal limit. So, the BekensteinHawking entropy (30) of the dilatonic $p$-brane (28) in $D$ dimensions has to be the same as the Bekenstein-Hawking entropy of a black hole solution (17) of the associated twodimensional dilaton gravity theory in the large charge limit or the near-extremal limit.

\section{NONEXTREME BLACK HOLES WITH REGULAR BPS LIMIT}

In this section, we consider black holes in string theories with regular BPS limits. Such black holes are fourdimensional black holes with four charges and fivedimensional black holes with three charges. The previous related works (e.g., Refs. [13-15]) relate such black holes to the two-dimensional charged black hole solution of McGuigan, Nappi, and Yost [21], which has a nontrivial dilaton field as well as a U(1) gauge field. In such works, black hole solutions which contain a charge associated with the gravitational wave are considered or U-duality transformations are applied to obtain solutions with the gravitational wave charge. Then, one takes the limit in which only the harmonic functions associated with other charges take the near-horizon limit form, while the harmonic function associated with the gravitational wave (and the harmonic function of the fundamental string with the charge assumed to be equal to the gravitational wave charge) does not take the near-horizon form. In this limit, the string-frame spacetime metric is put into the form of the direct product of the McGuigan-Nappi-Yost black hole and a sphere after the coordinate transformation. Then, the Bekenstein-Hawking entropy of the two-dimensional black hole becomes exactly the same as the Bekenstein-Hawking entropy of the $D=4,5$ black holes.

When one counts the microscopic degrees of freedom associated with the black hole entropy by making use of the two-dimensional model or the three-dimensional model of the BTZ black hole [10], one considers conformal theory at the spacetime boundary, which is associated with the gravity theory only and therefore does not have anything to do with string theories (although, it is shown [35-37] that the BTZ black hole solution can be embedded as a solution of string theory). To put it another way, the microscopic degrees of freedom in such a picture are not associated with the degenerate string states, whose density is invariant under the $U$-duality transformations. We also note that the form of the Einstein-frame spacetime metric, which gives rise to the Bekenstein-Hawking entropy formula, is insensitive to different ways of embedding black holes, such as the higherdimensional intersecting branes in string theories or $\mathrm{M}$ theory. So, it seems to be unnatural to uplift the black hole solutions to ten dimensions and apply a series of U-duality transformations to make the black hole solutions carry the charge associated specifically with the gravitational wave of string theory for the purpose of relating the near-horizon limit spacetime metric to the BTZ black hole solution (in spacetime in one higher dimensions) or to the twodimensional charged black hole solution of McGuigan, Nappi, and Yost. Also, it seems to be unnatural to let only part of the harmonic functions take near-horizon limit forms (by applying the series of U-duality transformations and the so-called shift transformation), while that associated with the gravitational wave (and fundamental string) is not taking the near-horizon limit form, in order to relate the $D=4,5$ black hole solutions to the BTZ black hole solution (and to the two-dimensional charge black hole of McGuigan, Nappi, and Yost), when we take notice of the fact that all the harmonic functions of the black hole solutions in the Einstein-frame are actually on equal footing (i.e., the Einstein-frame metric is symmetric under the permutations of harmonic functions). Such unnaturalness becomes pronounced for the particular case of the Reissner-Nordstrom black holes, i.e, the case of equal constituent charges. First, when all the charges are equal, all the scalars of the solutions are constant, but the equal charge limit of the above-mentioned near-horizon limit solutions does not lead to constant scalar fields since some of the harmonic functions have a near-horizon form and some do not. Second, it is unnatural to let some of the harmonic 
functions not take near-horizon forms, when all the charges have the same magnitude.

So, in this section, we consider only the generic Einsteinframe spacetime metric for black hole solutions, disregarding the higher-dimensional origin of constituent charges and taking all the harmonic functions on equal footing. We will, therefore, not uplift the $D=4,5$ black holes to higher dimensions and we will let all the harmonic functions associated with the constituent charges take the near-horizon limit forms. Perhaps, our description of a nonextreme black hole with the regular BPS limit in this section may not lead to the correct description of black hole thermodynamics. But it seems to be more natural from the perspective of the Einstein-frame form of the spacetime metric of black hole solutions. The generic property of stringy black holes with the regular BPS limit is that in the near-horizon limit all the scalar fields (including dilaton) of the black hole solutions become constant and the spacetime metric takes the $\mathrm{AdS}_{2} \times S^{n}$ form. Therefore, it seems that the solution of the associated two-dimensional model should have a constant dilaton field.

The most general action for the two-dimensional dilaton gravity, which depends at most on two derivatives of the fields, can be transformed to the following form [38,39]:

$$
S=\frac{1}{2 \kappa_{2}^{2}} \int d^{2} x \sqrt{-g}\left[\phi \mathcal{R}_{g}+V(\phi)\right] .
$$

The field equations of this action have a solution with constant dilaton $\phi=\phi_{0}$, provided that the potential $V(\phi)$ satisfies the following conditions [40]:

$$
V\left(\phi_{0}\right)=0,\left.\quad \frac{d V(\phi)}{d \phi}\right|_{\phi_{0}} \neq 0 .
$$

Then, in the conformal gauge with the following spacetime metric:

$$
g_{\mu \nu} d x^{\mu} d x^{\nu}=e^{2 \rho}\left(-d t^{2}+d x^{2}\right),
$$

the field equations of the action (34) for a static configuration

$$
\begin{aligned}
\frac{d^{2} \rho}{d x^{2}}+\frac{1}{2} e^{2 \rho} \frac{d V}{d \phi} & =0, \\
\frac{d^{2} \phi}{d x^{2}}-e^{2 \rho} V & =0,
\end{aligned}
$$

lead to the solution with constant spacetime curvature, i.e., $\phi=\phi_{0}$ and $\mathcal{R}_{g}=2 e^{-2 \rho\left(d^{2} \rho / d x^{2}\right)}=-V^{\prime}\left(\phi_{0}\right)$. This is in accordance with the fact that the near-horizon region spacetimes of the four-dimensional black hole with four charges and the five-dimensional black hole with three charges contain the $\mathrm{AdS}_{2}$ space. The following spacetime metric solution [40] to the field equations (37) in the Schwarzschild gauge is obtained by redefining the spatial coordinate through the relation $d y=e^{2 \rho} d x$ :

$$
d s_{2}^{2}=-\left(\frac{\mathcal{R}_{0}}{2} y^{2}-k\right) d t^{2}+\left(\frac{\mathcal{R}_{0}}{2} y^{2}-k\right)^{-1} d y^{2}
$$

where $\mathcal{R}_{0}=-V^{\prime}\left(\phi_{0}\right)$ is the Ricci scalar of the metric and $k$ is an integration constant.

In the following subsections, we bring the near-horizon region metrics of the $D=4,5$ black holes to the form of solution (38). Then, the study of the thermodynamics of nonextreme black holes in $D=4,5$ with a regular BPS limit reduces to the study of two-dimensional black holes with a constant dilaton field.

\section{A. Four-dimensional black hole}

The generic form of the Einstein-frame metric of the fourdimensional black hole solution with four charges is

$$
\begin{aligned}
g_{\mu \nu}^{E} d x^{\mu} d x^{\nu}= & -\frac{1}{\sqrt{H_{1} H_{2} H_{3} H_{4}}} f d t^{2} \\
& +\sqrt{H_{1} H_{2} H_{3} H_{4}}\left[f^{-1} d r^{2}+r^{2} d \Omega_{2}^{2}\right],
\end{aligned}
$$

where $f=1-m / r$ and $H_{i}=1+m \sinh ^{2} \alpha_{i} / r$. Here, the U(1) charges $Q_{i} \sim m \sinh 2 \alpha_{i}$ can have any higher-dimensional origin. Namely, the metric (39) can be the metric of a heterotic black hole solution [41-43] with the Kaluza-Klein U(1) electric and magnetic charges and the Neveu-SchwarzNeveu-Schwarz (NS-NS) 2-form U(1) electric and magnetic charges or the metric of a type-IIB black hole solution compactified from intersecting D3 branes. Regardless of various higher-dimensional origins as intersecting branes, the Einstein-frame metric for all the four-dimensional stringy black holes with four charges have the form (39).

In the near-horizon region, in which $H_{i} \approx m \sinh ^{2} \alpha_{i} / r(i$ $=1, \ldots, 4)$, the metric (39) is approximated to

$$
\begin{aligned}
g_{\mu \nu}^{E} d x^{\mu} d x^{\nu} \approx- & \frac{r^{2}}{m^{2} \prod_{i=1}^{4} \sinh \alpha_{i}}\left(1-\frac{m}{r}\right) d t^{2} \\
& +\frac{m^{2} \prod_{i=1}^{4} \sinh \alpha_{i}}{r^{2}}\left(1-\frac{m}{r}\right)^{-1} d r^{2} \\
& +m^{2} \prod_{i=1}^{4} \sinh \alpha_{i} d \Omega_{2}^{2} .
\end{aligned}
$$

The two-dimensional part of this near-horizon region metric can be put into the following suggestive form of the constant dilaton solution (38) of the two-dimensional dilaton gravity by redefining the spatial coordinate as $y=r-m / 2$ : 


$$
\begin{aligned}
d s_{2}^{2} \approx & -\left(\frac{y^{2}}{m^{2} \prod_{i=1}^{4} \sinh \alpha_{i}}-\frac{1}{4 \prod_{i=1}^{4} \sinh \alpha_{i}}\right) d t^{2} \\
& +\left(\frac{y^{2}}{m^{2} \prod_{i=1}^{4} \sinh \alpha_{i}}-\frac{1}{4 \prod_{i=1}^{4} \sinh \alpha_{i}}\right)^{-1} d y^{2} .
\end{aligned}
$$

This corresponds to the two-dimensional solution with a constant dilaton and constant spacetime curvature $\mathcal{R}_{g}=$ $-V^{\prime}\left(\phi_{0}\right)=2 /\left(m^{2} \Pi_{i=1}^{4} \sinh \alpha_{i}\right)$.

\section{B. Five-dimensional black hole}

The generic form of the Einstein-frame metric of the fivedimensional black hole solution in string theory with three charges, regardless of the higher-dimensional origins of the charges, is as follows:

$$
\begin{aligned}
g_{\mu \nu}^{E} d x^{\mu} d x^{\nu}= & -\frac{1}{\left(H_{1} H_{2} H_{3}\right)^{2 / 3}} f d t^{2} \\
& +\left(H_{1} H_{2} H_{3}\right)^{1 / 3}\left[f^{-1} d r^{2}+r^{2} d \Omega_{3}^{2}\right],
\end{aligned}
$$

where $f=1-m / r^{2}$ and $H_{i}=1+m \sinh ^{2} \alpha_{i} / r^{2}$. In the nearhorizon region, in which $H_{i} \approx m \sinh ^{2} \alpha_{i} / r^{2}(i=1,2,3)$, the metric (42) is approximated to

$$
\begin{aligned}
g_{\mu \nu}^{E} d x^{\mu} d x^{\nu} \approx- & \frac{r^{4}}{m^{2} \prod_{i=1}^{3} \sinh ^{4 / 3} \alpha_{i}}\left(1-\frac{m}{r^{2}}\right) d t^{2} \\
& +\frac{m \prod_{i=1}^{3} \sinh ^{2 / 3} \alpha_{i}}{r^{2}}\left(1-\frac{m}{r^{2}}\right)^{-1} d r^{2} \\
& +m \prod_{i=1}^{3} \sinh ^{2 / 3} \alpha_{i} d \Omega_{3}^{2} .
\end{aligned}
$$

The two-dimensional part of the metric (43) can be put into the following suggestive form of the two-dimensional constant dilaton solution (38) by redefining the spatial coordinate as $y=1 / 2 m^{1 / 2} \Pi_{i=1}^{3} \sinh ^{1 / 3} \alpha_{i}\left(r^{2}-m / 2\right)$ :

$$
\begin{aligned}
d s_{2}^{2} \approx & -\left(\frac{4 y^{2}}{m \prod_{i=1}^{3} \sinh ^{2 / 3} \alpha_{i}}-\frac{1}{4 \prod_{i=1}^{3} \sinh ^{4 / 3} \alpha_{i}}\right) d t^{2} \\
& +\left(\frac{4 y^{2}}{m \prod_{i=1}^{3} \sinh ^{2 / 3} \alpha_{i}}-\frac{1}{4 \prod_{i=1}^{3} \sinh ^{4 / 3} \alpha_{i}}\right)^{-1} d y^{2} .
\end{aligned}
$$

This corresponds to the two-dimensional solution with the constant dilaton and the constant spacetime curvature $\mathcal{R}_{g}=$ $-V^{\prime}\left(\phi_{0}\right)=8 /\left(m \Pi_{i=1}^{3} \sinh ^{2 / 3} \alpha_{i}\right)$.
[1] J. Polchinski, Phys. Rev. Lett. 75, 4724 (1995).

[2] A. Strominger and C. Vafa, Phys. Lett. B 379, 99 (1996).

[3] G. 't Hooft, "Dimensional reduction in quantum gravity,", gr-qc/9310026.

[4] L. Susskind, Phys. Rev. D 49, 6606 (1994).

[5] L. Susskind, J. Math. Phys. 36, 6377 (1995).

[6] J. D. Brown and M. Henneaux, Commun. Math. Phys. 104, 207 (1986).

[7] S. Carlip, Phys. Rev. D 51, 632 (1995).

[8] S. Carlip, Phys. Rev. D 55, 878 (1997).

[9] A. Strominger, J. High Energy Phys. 02, 009 (1998).

[10] M. Banados, C. Teitelboim, and J. Zanelli, Phys. Rev. Lett. 69, 1849 (1992).

[11] S. Hyun, " $U$-duality between three and higher dimensional black holes,' hep-th/9704005.

[12] K. Sfetsos and K. Skenderis, "Microscopic derivation of the Bekenstein-Hawking entropy formula for non-extremal black holes,' Nucl. Phys. B517, 179 (1998).

[13] E. Teo, Phys. Lett. B 430, 57 (1998).

[14] G. L. Cardoso, Phys. Lett. B 432, 65 (1998).

[15] G. L. Cardoso and T. Mohaupt, Phys. Lett. B 435, 277 (1998).

[16] Y. Kiem, C. Lee, and D. Park, Phys. Rev. D 58, 125002 (1998).

[17] M. Cadoni, Phys. Rev. D 60, 084016 (1999).
[18] R. Jackiw, in Quantum Theory of Gravity, edited by S. Christensen (Hilgar, Bristol, 1984), p. 403.

[19] C. Teitelboim, in Quantum Theory of Gravity (Ref. [18]), p. 327.

[20] C. G. Callan, S. B. Giddings, J. A. Harvey, and A. Strominger, Phys. Rev. D 45, 1005 (1992).

[21] M. D. McGuigan, C. R. Nappi, and S. A. Yost, Nucl. Phys. B375, 421 (1992).

[22] M. Cadoni and S. Mignemi, Phys. Rev. D 59, 081501 (1999).

[23] M. Cadoni and S. Mignemi, “Asymptotic symmetries of $\mathrm{AdS}_{2}$ and conformal group in $d=1$,' hep-th/9902040.

[24] J. Navarro-Salas and P. Navarro, " $\mathrm{AdS}_{2} / \mathrm{CFT}_{1}$ correspondence and near-extremal black hole entropy," hep-th/9910076.

[25] J. L. Cardy, Nucl. Phys. B270, 186 (1986).

[26] S. Carlip, Phys. Rev. Lett. 82, 2828 (1999).

[27] S. N. Solodukhin, Phys. Lett. B 454, 213 (1999).

[28] M. J. Duff and J. X. Lu, Nucl. Phys. B416, 301 (1994).

[29] G. W. Gibbons and P. K. Townsend, Phys. Rev. Lett. 71, 3754 (1993).

[30] M. J. Duff, G. W. Gibbons, and P. K. Townsend, Phys. Lett. B 332, 321 (1994).

[31] H. J. Boonstra, K. Skenderis, and P. K. Townsend, J. High Energy Phys. 01, 003 (1999).

[32] D. Youm, “(Generalized) conformal quantum mechanics of 
0-branes and two-dimensional dilaton gravity,' hep-th/9909180.

[33] D. Louis-Martinez and G. Kunstatter, Phys. Rev. D 49, 5227 (1994).

[34] R. B. Mann, Phys. Rev. D 47, 4438 (1993).

[35] G. T. Horowitz and D. L. Welch, Phys. Rev. Lett. 71, 328 (1993).

[36] N. Kaloper, Phys. Rev. D 48, 2598 (1993).

[37] A. Ali and A. Kumar, Mod. Phys. Lett. A 8, 2045 (1993).

[38] T. Banks and M. O’Loughlin, Nucl. Phys. B362, 649 (1991).
[39] D. Louis-Martinez, J. Gegenberg, and G. Kunstatter, Phys. Lett. B 321, 193 (1994).

[40] J. Cruz, A. Fabbri, D. J. Navarro, and J. Navarro-Salas, Phys. Rev. D 61, 024011 (2000).

[41] M. Cvetič and D. Youm, Phys. Rev. D 53, 584 (1996).

[42] M. Cvetic and D. Youm, "BPS saturated and non-extreme states in Abelian Kaluza-Klein theory and effective $\mathrm{N}=4$ supersymmetric string vacua,' hep-th/9508058.

[43] M. Cvetič and D. Youm, Nucl. Phys. B472, 249 (1996). 